\title{
On Taxation Pass-Through for a Monopoly Firm*
}

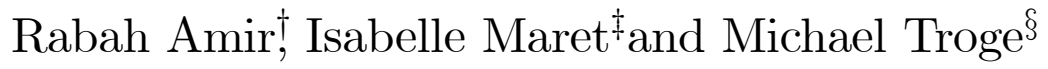

January 2004

\begin{abstract}
This paper investigates the pass-through of an excise tax imposed on a monopoly firm with constant marginal cost. The optimal price increases as tax increases for any demand function. Tax pass-through is globally under or in excess of $100 \%$ according as the direct demand function is log-concave or log-convex. The analysis relies on supermodular optimization and delivers conclusions based on minimal sufficient assumptions in a simple, broadly accessible and self-contained framework. Further results allow for mixed conditions that provide precise and local determination of pass-through. Several illustrative examples are given. Policy conclusions relating to the relative wisdom of taxing high versus low cost monopoly firms are drawn from the results.
\end{abstract}

${ }^{*}$ The authors would like to thank Jean Hindriks, Laurent Linnemer, Gwenael Piaser, John Roberts for some discussions or feedback on the general topic of this paper.

${ }^{\dagger}$ CORE and Department of Economics, University of Louvain, 1348 Louvain-la-Neuve, Belgium. (Email: amir@core.ucl.ac.be).

¥BETA, CNRS-UMR 7522, Université Louis Pasteur, 61 avenue de la Forêt-Noire, 67085 Strasbourg, France. (Email: maret@cournot.u-strasbg.fr)

§ESCP-EAP, European School of Management, 79 ave. de la République, 75543 Paris. 


\section{Introduction}

The theory of taxation has been part of the world of modern economics from en early stage (see e.g. Wicksell, 1896). Of the many strands of literature on the topic, one of the more active fields of research in recent years is the theory of taxation of firms in a partial equilibrium framework (Suits and Musgrave, 1953, Seade, 1985, Kimmel, 1992, Hamilton, 1999, and Anderson, de Palma and Kreider, 2001, among others.) The latter study provides in its introduction a detailed historical account of the importance of taxation in most economies, and we simply refer the reader there for a complete motivation of the economic importance of the topic at hand.

In this literature, the private and the public effects of taxation are investigated for various market structures. While the earlier studies typically dealt with monopoly and perfect competition, more recent work considered mostly oligopolistic competition. The incidence of taxation can be in the form of ad valorem or specific taxes, imposed either on the revenue side or the production side of the market.

The present paper deals with the effects of a per-unit unit tax on production levied on a monopoly firm with constant marginal cost facing a general market demand curve. The central issue raised in this paper is to determine the most general conditions under which the behavior of a monopoly firm in response to such a form of taxation can be fully characterized. As this issue has received quite a few treatments in the past, within various market structures, one of the first things that comes to mind is to justify why this problem deserves a new look at all. The main reason is that previous treatments have not explicitly sought to provide minimally sufficient conditions under which their conclusions are valid.

In view of the fact that the issue at hand is essentially a comparative statics problem, it is natural to approach it using the new, lattice-theoretic, methodology for handling such problems. By introducing the technique of supermodular optimization ${ }^{1}$

\footnotetext{
${ }^{1}$ This theory was developed by Topkis (1978), and further extended by Vives (1990), Milgrom and Roberts (1990), Milgrom and Shannon (1994) and Athey (2002). Applications to oligopoly that
} 
into this literature, we arrive at tight, simple and clear-cut conditions that validate the derived conclusions, most of which have less general antecedants. More specifically, by dispensing with the need for second-order conditions, a necessity if one uses the traditional methods based on the Implicit Function Theorem applied to the firstorder conditions, one arrives at sufficient conditions that are critical for the desired conclusion, and hence meaningfully interpretable from an economic point of view.

To illustrate these points clearly, let us provide a preview of our main results. Using price as the decision variable of the firm, it is shown that the optimal price will always increase with the tax rate, assuming no first or second-order properties on the direct demand function at all. Thus a monopoly firm will increase its optimal price in response to an increase in the tax rate even if demand is not globally decreasing, concave, or even continuous, provided the firm's profit maximization problem admits an optimal price. As to the question of assessing the size of the tax pass-through - i.e. how much price increases relative to the increase in the tax rate in percentage terms - the answer is that it is less than $100 \%$ if the direct demand function is log-concave and more than $100 \%$ if it is log-convex. As there is no explicit need for a secondorder condition that would be common to these two mutually exclusive cases, the underlying mutually exclusive assumptions fully explain the result from an intuitive point of view. The paper also provides some insight and results about some specific cases of local determination of the tax pass-through.

While the main contribution of the paper may be deemed more methodological, it remains nevertheless true that the derived results are quite interesting from an economic point of view as well. Several illustrative examples are given all along the paper that enhance the readability of the general analysis, and provide an immediate sense of the usefulness of the results. Some of these examples would violate the second-order conditions of most previous treatments of tax pass-through, and thus inspired the present paper are Amir (1996) and Amir and Lambson (2000). Topkis (1998) and Vives (1999) offer book-level treatments. 
would not quite fit the known results in a clear-cut manner.

While this paper is confined to the monopoly case, an extension of the analysis to oligopolistic competition would be of substantial interest for future research. The same approach is conjectured to be appropriate in that context as well, and will likely yield new insights into the issue at hand.

The paper is organized as follows. Section 2 provides a summary of the definitions and results from supermodular optimization that will be used in this paper, in an extremely simplified form that is sufficient for our purposes here. The results and the associated ilustrations are given in Section 3. A final section summarizes some aspects of policy relevance that may be drawn from the results.

\section{Mathematical Preliminaries}

Consider a parametrized family of optimization problems, where $S \subset R$ is a parameter set, $A_{s} \subset A \subset R$ (for some action set $A$ ) is the set of feasible actions when the parameter is $s$, and $F: S \times A \rightarrow R$ is the objective function:

$$
a(s)=\arg \max \left\{F(s, a): a \in A_{s}\right\} .
$$

The aim is to derive sufficient conditions on the objective and constraint set that yield monotone optimal argmax's.

A function $F: S \times A \rightarrow R$ is (strictly) supermodular ${ }^{2}$ in $(s, a)$ if $\forall a^{\prime}>a, s^{\prime}>s$

$$
F\left(s^{\prime}, a^{\prime}\right)-F\left(s^{\prime}, a\right)(>) \geq F\left(s, a^{\prime}\right)-F(s, a)
$$

or in other words if the difference $F\left(\cdot, a^{\prime}\right)-F(\cdot, a)$ is an increasing function ${ }^{3}$.

For smooth functions, supermodularity admits a convenient test (Topkis, 1978) ${ }^{4}$

\footnotetext{
${ }^{2}$ This is really the notion of increasing differences, which in $R^{2}$ is equivalent to supermodularity.

${ }^{3}$ Throughout, a function $f: S \rightarrow R$ is increasing (decreasing) if $x \geq y \Rightarrow f(x) \geq(\leq) f(y)$. It is strictly increasing (decreasing) if $x>y \Rightarrow f(x)>(<) f(y)$.

${ }^{4}$ Furthermore, if $\partial^{2} f(a, s) / \partial a \partial s>0$ then $F$ is strictly supermodular. On the other hand, the reverse implication need not hold.
} 
Lemma 1 If $F$ is twice continuously differentiable, supermodularity is equivalent to $\partial^{2} F(s, a) / \partial a \partial s \geq 0$, for all $a$ and $s$.

Supermodularity formalizes the usual notion of complementarity: Having more of one variable increases the marginal returns to having more of the other variable.

A simplified version of Topkis's (1978) Monotonicity Theorem is now given. It is assumed throughout that $F$ is continuous (or even just upper semi-continuous) in $a$ for each $s$, so that the max in (1) is attained. Furthermore, the correspondence $a(s)$ then admits maximal and minimal selections, denoted $\bar{a}(s)$ and $\underline{a}(s)$ respectively.

Theorem 2 Assume that

(i) $F$ is supermodular in $(s, a)$, and

(ii) $A_{s}=[g(s), h(s)]$ where $h, g: S \rightarrow R$ are increasing functions with $g \leq h$.

Then the maximal and minimal selections of $a(s), \bar{a}(s)$ and $\underline{a}(s)$, are increasing functions. Furthermore, if (i) is strict, then every selection of a $(s)$ is increasing.

Sometimes, one might be interested in having a strictly increasing argmax.

Theorem 3 Assume $F$ is continuously differentiable, $\partial F / \partial a$ is strictly increasing in $s$ and the argmax is interior. Then every selection of a $(s)$ is strictly increasing.

Since supermodularity is equivalent to $\partial F / \partial a$ being increasing in $s$, the assumption in Theorem 3 is a minor strengthening of the supermodularity of $F$ (see Amir, 1996 or Edlin and Shannon, 1997 or Topkis, 1998 p.71 for a proof and further details.)

There are order-dual versions to all the above results. We state just the main one, giving obvious dual conditions under which an argmax is decreasing in the parameter. A function $F: S \times A \rightarrow R$ is (strictly) submodular if $-F$ is supermodular, i.e. if (2) holds with the inequality reversed.

Theorem 4 Assume that

(i) F is submodular in $(s, a)$, and 
(ii) $A_{s}=[g(s), h(s)]$ where $h, g: S \rightarrow R$ are decreasing functions with $g \leq h$.

Then the maximal and minimal selections of a $(s)$ are decreasing functions. Furthermore, if (i) is strict, then every selection of a $(s)$ is decreasing.

We say that a function $G: R_{+} \longrightarrow R_{+}$is log-concave (log-convex) if $\log G$ is concave (convex). The corresponding strict notions are defined in the obvious way. The following is a common way for supermodularity to arise.

Lemma 5 A function $G: R_{+} \longrightarrow R_{+}$is log-concave (log-convex) if and only if $G(x+y)$ is log-submodular (log-supermodular) in $(x, y)$.

For a smooth function $G: R_{+} \longrightarrow R_{+}$, log-concavity (log-convexity) is easily checked to be equivalent to

$$
G(x) G^{\prime \prime}(x)-\left[G^{\prime}(x)\right]^{2} \leq(\geq) 0 \text { for all } x
$$

The corresponding strict notions are given by (3) with a strict inequality.

\section{Set-up and Results on Pass-Through}

In this section, the problem at hand, dealing with the effects of taxation on optimal price and profit, is laid out and analyzed. Global results are stated and proved in the first subsection while results with some local flavor appear in the second subsection.

Consider a monopoly firm with constant unit cost $c$, charging a price $p \geq 0$, facing a tax $t$ per unit output, and operating in a market with a direct demand function $D:[0, \infty) \longrightarrow[0, \infty)$. The profit function is then (with $c$ viewed as a fixed parameter and $t$ as a variable parameter)

$$
(p, t)=(p-c-t) D(p)
$$

The issues at hand may alternatively be approached from the other well-known and equivalent formulation of the monopoly problem, namely, with $P(\cdot)$ denoting the 
inverse demand function (i.e. $P=D^{1}$ ), to choose an output level $q$ so as to

$$
\max \{\pi(q, t)=q P(q)-(c+t) q: q \geq 0\}
$$

It turns out that an analysis based on price as the decision variable is more natural here, as our interest is mostly in the comparative statics property of the optimal price.

As the price choices in $[0, c+t)$ are clearly dominated by the choice of $p=c+t$, we may, and often will find it convenient, to restrict prices to the set $p \in[c+t, \infty)$.

Without further mention, the following assumption will be maintained (for convenience) throughout this paper:

(A1) The demand function $D$ is a continuous function.

With $c$ fixed, denote optimal profit for a given tax level by

$$
(t)=\max \{(p, t): p \geq c+t\}
$$

\subsection{Results of a Global Nature}

The results here are extensions or generalizations of existing results in the literature. The added generality is achieved via our use of supermodularity techniques, and is manifested in the abscence of customary assumptions of interiority of solutions, smoothness of the model functions, and second-order type conditions (i.e. some form of concavity of the profit function.)

Our first result deals with the dependence of optimal profit on the tax rate.

Proposition 6 Under Assumption (A1) alone, $\quad(t)$ is a decreasing convex function.

Proof. Let $t^{\prime}>t$. Since $\left(p, t^{\prime}\right) \leq(p, t)$ and the feasible sets are such that $\left[c+t^{\prime}, \infty\right) \subset[c+t, \infty)$, it follows that $(t) \geq\left(t^{\prime}\right)$. Hence $(t)$ is decreasing in $t$.

To show convexity, observe that $(t)$ is the pointwise supremum of an (uncountable) family of affine functions in $t$. It follows directly from a well-known result in convex analysis (Rockaefellar, 1970, p. 35) that $(t)$ is convex. 
Thus the marginal decline in a monopoly's profit due to one extra unit in the tax rate decreases with the firm's cost. Another economic implication of this result is that a firm would always prefer a lottery on the tax rate to the sure incidence of the expected value of that lottery.

As the optimal price is invariant under a strictly monotonic transformation, we may equivalently consider the objective

$$
\log (p, t)=\log (p-c-t)+\log D(p), p \in[c+t, \infty)
$$

Denote the optimal price correspondence by $p(t)$ throughout (we omit the dependence on $c$ for simplicity, it being understood that the variable parameter below is $t$.)

Proposition 7 Under Assumption (A1) and the additional assumption that $D(c+$ $t)>0$, for all $t>0$, and $p D(p) \longrightarrow 0$ as $p \longrightarrow \infty$, every selection from the optimal price $p(t)$ is strictly increasing in $t$.

Proof. From the assumption that $D(c+t)>0$, for all $t>0$, and $p D(p) \longrightarrow 0$ as $p \longrightarrow \infty$, it follows that there exists $\bar{p} \in(c+t,+\infty)$ such that $(\bar{p}, t)>0$ and $\lim _{p \rightarrow+\infty}(p, t) \leq 0$ for all $t$. Since, $\quad(c+t, t)=0$, there is an interior price argmax

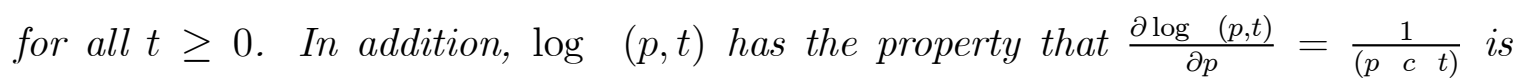
strictly increasing in $t$, therefore the conclusion follows directly from Theorem 3.

While a version of this result is broadly known, observe that the present statement is stronger than usual, in that the demand function need not be decreasing or even continuous in own price for this conclusion to hold ${ }^{5}$ ! Furthermore, no second-order condition is needed either here, whereas standard treatments will invariably assume some sort of concavity condition on the demand or the profit functions. The latter are often violated by some commonly used demand functions, such as those of the hyperbolic family, and constitute thus rather restrictive assumptions for deriving the

\footnotetext{
${ }^{5}$ All that is really needed is for $D$ to be upper semi-continuous in $p$ instead of Assumption (A1), so that maximizing profit amounts to maximizing an upper semi-continuous function on a compact set, and then invoking Weirstrass's classical theorem.
} 
natural conclusion that a higher tax will lead to a higher price by a monopoly firm, which as demonstrated by Proposition 7, is an essentially universal result.

Due to the abscence of a second-order condition, there may be no selection of the optimal price correspondence that is a continuous function ot $t$ here, so that standard comparative statics methods relying on the implicit function theorem and first-order conditions are not applicable to derive Proposition 7 in the present level of generality. This result provides a simple illustration of the fact that the supermodularity approach typically yields comparative statics conclusions that rely only on critically needed assumptions on the primitives of a model. ${ }^{6}$

It follows directly from this result that the extremal selections from the optimal output correspondence are decreasing in $t$, a conclusion that can also be obtained by invoking Topkis's Theorem directly on the output formulation of the monopoly problem since the profit function in $(5)$ is submodular in $(q, t)$ for any demand function.

We now investigate further the upward reaction of price to an increase in the tax rate by comparing the size of the former to the size of the latter. To this end, a convenient change of variable will allow a very simple treatment of the question. Define mark-up (over the sum of marginal cost and tax) as $m \triangleq p-c-t$, and write the equivalent objective with this change of variable as

$$
\sim(m, t) \triangleq m D(m+c+t), \text { with } m=p-c-t
$$

As before, we will also need to consider the alternative objective

$$
\log \sim(m, t) \triangleq \log (m)+\log D(m+c+t) .
$$

The first result provides a sufficient condition for tax pass-through to be below $100 \%$.

\footnotetext{
${ }^{6}$ Here, the customary assumptions of continuity, monotonicity and concavity of the demand functions are superfluous conditions, the need for which arises from using the inadequate methodology of the implicit function theorem. As a consequence, such assumptions might interfere with an attempt to provide a precise economic interpretation for the result at hand, in view of the fact that they do not constitute critical limitations to the economic environment, which drive the comparative statics conclusion. Similar remarks will apply to other results derived in this paper.
} 
Proposition 8 If $D$ is log-concave, then there is a unique optimal price $p(t)$ for each $t$, and it satisfies $0<\frac{p\left(t^{\prime}\right) p(t)}{t^{\prime} t} \leq 1$ for all $t^{\prime}>t$, and is thus Lipschitz continuous.

Proof. Since $D$ is log-concave, $\log \sim(m, c)$ is strictly concave in $m$ (being the sum of a strictly concave function and a concave function). It follows that $m(t)$ is single-valued and continuous. Hence so is $p(t)$. As $m(t) \triangleq p(t)-c-t$, it follows that $p(t)$ has all slopes $\leq 1$ if and only if $m(t)$ is decreasing. We now establish the latter conclusion.

By Lemma 5, $D(m+c+t)$ is log-submodular in $(m, t)$ and so is $(m, t)$. Since the constraint on $m,[0, \infty)$, is constant in $t$, we conclude from Theorem 4 that $m(t)$ is globally decreasing in $t$. Since $m(t)=p(t)-c-t$, this is equivalent to the fact that $\frac{p\left(t^{\prime}\right) p(t)}{t^{\prime} t} \leq 1$. The overall conclusion (including Lipschitz continuity) follows by combining the latter slope condition with the fact that from Proposition 7 , we know that $0<\frac{p\left(t^{\prime}\right) p(t)}{t^{\prime} t}$.

Overall then, with log-concave demand, there is always positive but partial passthrough. A discussion of the level of generality of this proposition is postponed until the end of the statement of the results, although the reader unfamiliar with the notions of log-concavity might find it worthwhile to read the discussion first.

The next result strengthnens log-concavity of demand, i.e. $D D^{\prime \prime}-D^{\prime 2} \leq 0$ or $\frac{D^{\prime}(\cdot)}{D(\cdot)}$ being a decreasing function, to the strict version of the latter condition, and obtains a strict Lipschitz condition on the optimal price, as well as some handle on its interiority.

Proposition 9 Assume that $D$ is continuously differentiable on $[0, \infty)$, and that

(i) $D(c+t)>0$ for some $t>0$, and $p D(p) \longrightarrow 0$ as $p \longrightarrow \infty$.

(ii) $\frac{D^{\prime}(\cdot)}{D(\cdot)}$ is a strictly decreasing function.

Then there is a unique optimal price for each $t$, and some $\bar{t}$ such that $0<$ $\frac{p\left(t^{\prime}\right) p(t)}{t^{\prime} t}<1$ for all $t<t^{\prime} \leq \bar{t}$ and $p(t)=c+t$, for $t \geq \bar{t}$.

Proof. From the assumption in (i), it follows that marginal revenue $M R(p)=$ $D(p)+(p-c-t) D^{\prime}(p)$ satisfies $M R(c+t)>0$ (when $t$ is small) and $M R(p)<0$ for $p$ 
large enough. Hence, there is an interior price argmax, at least for sufficiently small values of $t$ (for a given $c$.) Uniqueness of the optimal price holds from Proposition 8 since (ii) is stronger than log-concavity of $D$ (as explained in the sentence preceding Proposition 9.)

Since $\frac{\partial \log ^{\sim}(m, t)}{\partial m}=\frac{D^{\prime}(m+c+t)}{D(m+c+t)}$ is strictly decreasing in $t$, it follows from the obvious dual to Theorem 3 that, whenever interior, $m(t)$ is strictly decreasing in $t$.

Since $m(t)=p(t)-c-t$, this is equivalent to the fact that $\frac{p\left(t^{\prime}\right) p(t)}{t^{\prime} t}<1$ for all $t^{\prime}, t$ such that $p(t)$ is interior.

However, as $t$ increases, there will come a point where $p(t)$ can no longer remain interior (i.e. $>c+t$ ), due to the fact that demand is fixed here (so that by assumption (i) of the Proposition, profits would eventually turn negative.) So for large enough $t$, we must have $D(c+t)=0$ and hence $p(t)=c+t$.

As the 0 bound on the slopes of $p(t)$ follows from previous results, the proof is now complete.

The next result provides a sufficient condition for tax pass-through to exceed $100 \%$ in a global sense.

Proposition 10 (a) If $D$ is log-convex, then the extremal selections from the optimal price $p(t)$ satisfy $\frac{p\left(t^{\prime}\right) p(t)}{t^{\prime} t} \geq 1$, for all $t^{\prime}>t$.

(b) If $\frac{D^{\prime}(\cdot)}{D(\cdot)}$ is a strictly increasing function, then all the selections from the optimal price $p(t)$ satisfy $\frac{p\left(t^{\prime}\right) p(t)}{t^{\prime} t}>1$ for all $t^{\prime}>t$ whenever $p(t)$ is interior.

Proof. (a) The objective function in (8) is supermodular in $(m, c)$ if $D$ is logconvex, by Lemma 5 . Since the constraint set, $m \in[0, \infty)$ is of the form specified in Theorem 2, it follows from the latter result that the maximal and minimal selections from the optimal mark-up $m(t)$ are increasing in $t$, or equivalently that the extremal selections of $p(t)$ have all their slopes $\geq 1$.

(b) When log-convexity is strengthened to $\frac{D^{\prime}(\cdot)}{D(\cdot)}$ being a strictly increasing function, the conclusion in (a) is strengthened to hold in a strict sense, whenever $p(t)$ is interior, for all possible selections, as follows directly from Theorem 3. 
We now discuss the scope of these results. The assumption of log-concavity is only mildly restrictive for a direct demand function ${ }^{7}$. Assuming smoothness, it is easier to grasp the level of generality of this assumption. As log-concavity is equivalent to (see Lemma 1)

$$
\partial^{2} \log D(m+c) / \partial m \partial c=\left[D D^{\prime \prime}-D^{\prime 2}\right] / D^{2} \leq 0 \text { or } D D^{\prime \prime}-D^{\prime 2} \leq 0
$$

it is clearly sufficient to have demand being concave or even not too convex. Most demand functions used in industrial economics satisfy log-concavity, including linear demand $D(p)=a-b p$, quadratic demand $D(p)=a-b p^{2}$, etc... The limiting case is $D(p)=e^{p}$, which is both convex and log-linear, thus also both log-concave and log-convex. An important class of demand functions that is excluded by logconcavity is the family of hyperbolic demands, including the well-known iso-elastic demand $D(p)=1 / p^{\alpha}, \alpha>1$. These functions are, on the other hand, log-convex. This property is rather restrictive for a demand function, as it requires a strong form of convexity ( $D^{\prime \prime}$ must be sufficiently positive so as to make $\left.D D^{\prime \prime}-D^{\prime 2} \geq 0\right){ }^{8}$

As illustration of these results, consider the following widely used examples in industrial organization. The first example has a log-concave demand.

Example 11 Consider the standard linear demand: $D(p)=a-p$ for $p \leq a$ and 0 for $p \geq a$, which is clearly concave, and thus log-concave. Then $(p, t)=(p-c-t)(a-p)$.

\footnotetext{
${ }^{7}$ It first appeared in Amir (1996) as the most general assumption on the inverse demand function that guarantees that the reaction correspondence of a firm in Cournot competition is downwardsloping, irrespective of the curvature properties of its cost function. See also Novshek (1985) for an alternative condition.

${ }^{8}$ There are some other hidden implications of log-concavity and log-convexity that are worthwhile to point out here. A necessary condition for log-convexity of a downward-sloping demand is that $D(p)$ be $>0$ for all $p \geq 0$, i.e that the function never touch the $x$-axis (see Amir, 1996 for justifications). Similarly, for demand functions a priori defined on $(0, \infty)$, such as isoelastic demands, log-concavity requires that $D(0)$ be finite.
} 
A standard calculation yields

$$
p(t)=\left\{\begin{array}{c}
(a+c+t) / 2 \text { if } c+t \leq a \\
c+t \text { if } c+t \geq a
\end{array} \text { and } p^{\prime}(t)=\left\{\begin{array}{c}
1 / 2 \text { if } c+t \leq a \\
1 \text { if } c+t \geq a
\end{array}\right.\right.
$$

So tax pass-through is $50 \%$ here, independently of the value of c.

The second example has a log-convex demand.

Example 12 Isoelastic demand: $D(p)=\frac{1}{p^{\alpha}}, \alpha>1, p \geq 0$.

As is easily checked, the elasticity of demand, defined in general as $\in \triangleq \frac{p}{q} \frac{d D}{d p}$, is here equal to $-\alpha$, a negative constant independent of the output level.

Here $(p, t)=\frac{\left(\begin{array}{ll}p & c\end{array}\right)}{p^{\alpha}}$.

A standard calculation shows that

$$
p(t)=\frac{c+t}{1-(1 / \alpha)} \text { and } \frac{d p(t)}{d t}=\frac{\alpha}{\alpha-1}=\frac{\varepsilon}{1+\varepsilon}>1, \text { for all } t .
$$

Hence, pass-through is again constant, but above $100 \%$.

These examples are well-known (see for instance Varian, 1992 p. 237). Propositions 8 and 10 are to be regarded then as natural generalizations of these particular illustrations, respectively.

The following example lies at the intersection of Propositions 8 and 10. It is obviously not a commonly used demand function, although for all relevant purposes for the issue at hand, it lies in the intermediate range between the previous commonly used examples.

Example 13 Let $D(p)=e^{p}, p \geq 0$.

As this demand is log-linear, thus both log-concave and log-convex, Propositions 8 and 10 imply that $\frac{d p}{d t}$ is $\leq 1$ and $\geq 1$, respectively. Hence we should have $\frac{d p}{d t}=1$.

Here $(p, t)=(p-c-t) e^{p}$. The first-order condition reduces to $e^{p}-(p-c-$ t)e ${ }^{p}=0$, so that, as expected,

$$
p(t)=c+t+1 \text { and } \frac{d p(t)}{d t}=1
$$


As to the economic intuition behind the results on the size of pass-through, it is quite staightforward. Recall that an increase in the tax rate always leads to an output reduction (see (5) and the discussion below it). When demand is log-convex, thus strongly convex, the output reduction translates into a price increase at an increasing rate, whence the more than $100 \%$ pass-through. In the presence of several Cournot competitors, there is a stronger version of this result owing to the underlying strategic effects (see e.g. Seade, 1985.)

\subsection{Results with a Local Flavor}

To recapitulate, Proposition 7 tells us that the price pass-through of an excise tax is always positive, and Propositions 8 and 10 provide respective sufficient global conditions on the direct demand function under which pass-through is below or above 100\%. Nevertheless, as lots of plausible demand functions are neither globally logconcave nor globally log-convex, the results so far do obviously not capture the full scope of possible behavior of tax pass-through. Put differently, there are many reasonable demand functions for which tax pass-through may change from being less than, to being more than, $100 \%$, depending on the level of the firm's marginal cost $c$. We now investigate such hybrid cases and present a general result dealing with the varying size of pass-through.

To this end, we begin with an observation to the effect that Proposition 8 does not provide minimally sufficient conditions for its conclusion. To illustrate this point, consider the following example.

Example 14 Let $D(p)=-\log p$ for $p \leq 1$ and 0 for $p \geq 1$.

The profit function is $(p, t)=-(p-c-t) \log p$, with $c+t \leq 1$.

To check log-concavity/convexity, we compute, by direct differentiation,

$$
D^{\prime}(p)=-\frac{1}{p}, D^{\prime \prime}(p)=\frac{1}{p^{2}} \text {, and } D D^{\prime \prime}-D^{\prime 2}=-\frac{1}{p^{2}}(\log p+1) .
$$


Hence,

$$
D D^{\prime \prime}-D^{\prime 2} \lesseqgtr 0 \text { according as } p \gtreqless e^{1} \approx 0.368 \text {. }
$$

Hence, $D$ is log-convex for $p \in\left[0, e^{1}\right]$ and log-concave for $p \in\left[e^{1}, \infty\right)$.

We now compute the optimal price directly. The first-order condition is

$$
-\log p-\frac{(p-c-t)}{p}=0
$$

As no closed-form solution is possible, implicit differentiation yields

$$
p^{\prime}(t)=\frac{p}{p+c+t}, \text { so that } p^{\prime}(t)<1 \text {. }
$$

This establishes that the conclusion of Proposition 8 holds although the concomitant assumption - log-concavity of D - fails to hold globally (cf. (9)), i.e. on the set $\{(p, c, t): 0 \leq p \leq 1$ and $c+t \leq p\}$.

It turns out that a variant of Proposition 8 will have a bearing on this example (see the next proposition.) To provide a preview, first observe that it follows from (10) that the optimal monopoly price corresponding to $c=t=0$ is such that $-\log p(0)-1=0$, or $p(0)=e^{1} \approx .368$. In view of Proposition 7 , we know that as $t$ is increased from 0 (with $c$ still $=0), p(t)>p(0)=e^{1}$, for all $t>0$, with this conclusion being independent of any assumptions on $D$ ! Indeed, (9) shows that $D$ here is log-concave on $\left[e^{1}, 1\right]$, which is all that is needed for the conclusion that $p^{\prime}(t) \leq 1$. For the stronger conclusion that $p^{\prime}(t)<1$, one needs to apply the same reasoning to the dual of Theorem 3, an extension that is not pursued any further below.

This motivates the following generalization of Proposition 8.

Proposition 15 Let $p(0)$ denote the optimal monopoly price when $t=0$, with $c$ fixed. If $D$ is log-concave on $[p(0), \infty)$, then there is a unique optimal price $p(t)$ for all $t>0$, and it satisfies $0<\frac{p\left(t^{\prime}\right) p(t)}{t^{\prime} t} \leq 1$ for all $t^{\prime}>t>0$.

Proof. By Proposition 7, we know that $p(t)>p(0)$ for all $t>0$. Hence, $m(t)=p(t)-t-c \geq p(0)-t-c$. So $m(t) \subseteq[p(0)-t-c, \infty)$ for all $t \geq$ 
0. Hence, the firm's best-response problem in (7) may be equivalently restated as $\max \{m D(m+t): m \in[p(0)-t-c, \infty)\}$. Since $D$ is log-concave on $[p(0), \infty), \log { }^{\sim}(m, t)$ is strictly concave in $m \in[p(0)-t-c, \infty$ ) (being the sum of a strictly concave function and a concave function). Hence, there is a unique optimal price $p(t)$ for all $t>0$.

Since $D$ is log-concave on $[p(0), \infty)$, the objective function $\log \sim(m, t)$ is $\log$ submodular in $(t, m)$ on the set $\{(t, m): t \geq 0$ and $m \in[p(0)-t-c, \infty)\}$. Furthermore, the feasible set $[p(0)-t-c, \infty)$ is obviously descending in $t$. Hence, by Theorem $4, m(t)$ is decreasing in $t$, or equivalently $\frac{p\left(t^{\prime}\right) p(t)}{t^{\prime} t} \leq 1$ for all $t^{\prime}>t>0$. The 0 bound on slopes follows from previous results.

Interestingly, the bound $e^{1}$ in Example 14 is tight and captures the full force of Proposition 15 as an improvement over Proposition 8, in that the set of prices for which log-concavity of demand fails to hold coincides exactly with the set of prices lower than the optimal price of the zero-cost monopoly. As it turns out, although demand is log-convex for small prices and log-concave for large prices, Example 14 does not really represent a hybrid case, due to the fact that not all possible prices are reached along an optimal behavior path for the monopolist, generated by varying $t$, as a consequence of Proposition 7, which holds for all (upper semi-continuous) demand functions. Only those prices above $p(0)$ will ever be reached, so that any binding restriction on demand need only be imposed on such prices.

We now provide a result dealing with actual hybrid cases.

Proposition 16 Suppose that there exists some $\widetilde{p}>p(0)$ such that $D$ is log-convex on $[0, \widetilde{p})$ and log-concave on $[\widetilde{p}, \infty)$. Then there exists a $\widetilde{t}$ such that the extremal selections of $p(t)$ satisfy $\frac{p\left(t^{\prime}\right) p(t)}{t^{\prime} t} \geq 1$ for all $t<t^{\prime}<\widetilde{t}$ and $0<\frac{p\left(t^{\prime}\right) p(t)}{t^{\prime} t} \leq 1$ for all $t^{\prime}>t>\widetilde{t}$

Proof. From Proposition 7, we know that all the selections of $p(t)$ are always strictly increasing globally, regardless of any curvature properties of $D$. Let $\widetilde{t}$ be the largest value of $t$ satisfying $p(\widetilde{t}) \leq \widetilde{p} \leq p\left(\widetilde{t}^{+}\right)$(note that this definition of $\widetilde{t}$ 
reflects the fact that if $p(t)$ is continuous at $\widetilde{t}$, then $\widetilde{t}$ is thereby simply defined by $p(\widetilde{t})=\widetilde{p})$. By the assumption of the proposition on $D$, we conclude that $D(m+t+c)$ is log-supermodular in $(m, t) \in\{(m, t): m+t+c \leq \widetilde{p}\}$ and log-submodular on $\{(m, t): \widetilde{p} \leq m+t+c<\infty\}$. The proof now follows by invoking, for the profit maximization problem, Proposition 10 for $(t, p) \in[0, \widetilde{t}] \times[t+c, \widetilde{p}]$ and Proposition 8 for $(t, p) \in[\widetilde{t}, \infty] \times[\widetilde{p}, \infty]$.

We next provide an illustrative example of a hybrid case that closely fits this Proposition.

Example 17 Let $D(p)=\log ^{2} p$ for $0<p \leq 1$ and 0 for $p \geq 1$, and $c=0$.

While this demand function might appear unusual, it does give rise to a wellbehaved profit function that is quasi-concave in price. Note also that as long as $0<$ $p \leq 1$, this demand is indeed downward-sloping, and that the profit function is bounded at $p=0$, although demand is not (see below.) The verification details of these points are left out for the sake of brevity.

To check that this demand is neither globally log-concave nor globally log-convex, direct differentiation yields

$$
D^{\prime}(p)=\frac{2}{p} \log p, D^{\prime \prime}(p)=\frac{2}{p^{2}}(1-\log p), \text { and } D D^{\prime \prime}-D^{\prime 2}=-\frac{2 \log ^{2} p}{p^{2}}(\log p+1) .
$$

Hence, as in Example 14,

$$
D D^{\prime \prime}-D^{\prime 2} \lesseqgtr 0 \text { according as } p \gtreqless e^{1} \approx 0.368 \text {. }
$$

We now compute the optimal price directly. The profit function is

$$
(p, t)=(p-t) \log ^{2} p, \text { with } t \leq p \leq 1
$$

The first-order condition reduces to ${ }^{9}$

$$
\log p+2 \frac{(p-t)}{p}=0 .
$$

\footnotetext{
${ }^{9}$ There is also another solution of the first-order condition at $p=1$, which turns out to be a local minimum, and is thus ignored w.l.o.g.
} 
Hence, $p(0)=e^{2}$. As no closed-form solution is possible here, implicit differentiation yields upon simplification

$$
p^{\prime}(t)=\frac{2 p}{p+2 t} .
$$

While this does not allow a direct conclusion as in Example 14, it follows from (13) that $p^{\prime}(0)=2 e^{2} / e^{2}=2$, so that $p^{\prime}(t)$ is not globally $<1$. In fact, it can be shown numerically (with details omitted for brevity) that

$$
p^{\prime}(t) \gtreqless 1 \text { according as } t \lesseqgtr 0.182 \text {. }
$$

Overall then, this example is a neat illustration of Proposition 15, with the following identification of the relevant constants: $\widetilde{p}=e^{1} \approx 0.368$ and $\widetilde{t} \approx 0.182$.

Finally, we state a result concerning a hybrid case with a demand function that is log-concave for low prices and log-convex for high prices.

Proposition 18 Suppose that there exists some $\widetilde{p}>p(0)$ such that $D$ is log-concave on $[0, \widetilde{p})$ and log-convex on $[\widetilde{p}, \infty)$. Then there exists a $\widetilde{t}$ such that the extremal selections of $p(t)$ satisfy $0<\frac{p\left(t^{\prime}\right) p(t)}{t^{\prime} t} \leq 1$ for all $t<t^{\prime}<\tilde{t}$ and $\frac{p\left(t^{\prime}\right) p(t)}{t^{\prime} t} \geq 1$ for all $t^{\prime}>t>\widetilde{t}$

The proof is left out as it is similar to the proof of the previous proposition.

\section{A Policy-Oriented Discussion and Conclusion}

We now provide a discussion of some simple policy implications of the above results. It is well-known that an excise tax is a generally undesirable way of taxing firms in comparison say to a lump-sum tax, in view of the distortive effects the former necessarily generates. In fact, this may be considered as a general principle of taxation. Yet at the same time, per-unit taxes on production are relatively common, in particular as a way of regulating pollution-producing firms, as these are situations in which the concomitant output decline engenders an environmental benefit. 
To the extent that excise taxes are used as policy tools on a regular basis, it is important to understand their relative effects, in as much detail as possible. In particular, regulators ought to be fully informed about the consequences of this form of taxation on consumer welfare. The results presented in this paper allow in particular for a better handle on the effects of such taxes on profits and on consumer welfare, as a function of the firm's current cost structure, which may be a reflection of the technological state of the firm.

While firms are always hurt by the imposition of such taxes, Proposition 6 also provides the conclusion that profits fall at a decreasing rate with the current unit cost of the firm. In other words, high-cost or small firms tend to suffer less than low-cost or large firms from a given unit tax increase (in absolute terms.)

As to the effect on consumer surplus, it is naturally always negative as price necessarily ends up moving upward (Proposition 7). Furthermore, it is also of interest to learn that this detrimental effect is worse when the market direct demand is logconvex than when it is log-concave, since tax pass-through exceeds $100 \%$ in the former case, while it is below $100 \%$ in the latter case.

In so-called hybrid cases, where the demand function changes curvature, going from an initial log-convex part to a log-concave part, tax pass-through is more than $100 \%$ for small values of the firm's unit cost (or relatively efficient firms) and less than $100 \%$ for high values of the unit cost (or inefficient firms). For demand functions having the reverse shape configuration, the result is simply accordingly reversed.

One can therefore draw some interesting conclusions in terms of the relative appropriateness of this form of taxation depending on the demand characteristics of industries. In cases with a demand function that is log-convex initially (as in Proposition 16), consumers will be prejudiced relatively more in case the tax incidence applies to an efficient firm than if it applies to an inefficient firm. ${ }^{10}$ Given the focus

\footnotetext{
${ }^{10}$ This result is reminiscent of the Boiteux-Ramsey taxation principle, which essentially states that commodities with low elasticities of substitution can be more effectively taxed than commodities with higher elasticities of substitution.
} 
of the present paper to the case of a monopoly firm, the above policy conclusion is not a priori applicable to two efficiency-differentiated firms operating in the same industry, unless we ignore all the strategic effects. The same conclusion is however applicable to two efficiency-differentiated firms operating in two different industries, if the regulatory authority comtemplates possibly taxing only one of them.

Interestingly, this conclusion gives rise to a testable hypothesis for which data ought to be relatively easy to collect. Within comparable industries in which general features about the shape of the demand function are known, is it often the case that firms of a given size are taxed more frequently than firms of the alternative size, according to the above theoretical conclusion?

By limiting consideration to monopoly firms, this analysis has ignored strategic effects in the market, which are known to be important co-determinants of the effects of taxation on profits and consumer surplus (see Seade, 1985 and Kimmel, 1992.) This remains a topic for future research along the same methodological lines. Of particular interest is to investigate whether the aforementioned conclusion about efficiencydifferentiated taxation carries through to an oligopoly setting with explicit strategic interaction..

\section{References}

[1] Amir, R. (1996a), "Cournot Oligopoly and the Theory of Supermodular Games", Games and Economic Behavior, 15, 132-148.

[2] Amir, R. (1996b), "Sensitivity Analysis in Multisector Optimal Economic Dynamics", Journal of Mathematical Economics, 25, 123-141.

[3] Amir, R. and V. Lambson (2000), "On the Effects of Entry in Cournot Markets", Review of Economic Studies, 67, 235-254. 
[4] Anderson, S., A. de Palma and B. Kreider (2001), "Tax Incidence in Differentiated Product Oligopoly", Journal of Public Economics, 81, 173-192.

[5] Athey, S. (2002), "Monotone Comparative Statics Under Uncertainty", Quarterly Journal of Economics, 117, 187-223.

[6] Edlin, A. and C. Shannon (1997), "Strict Monotonicity in Comparative Statics", Journal of Economic Theory, 89, 201-219.

[7] Hamilton, S. (1999), ", "Tax Incidence under Oligopoly: A Comparison of Policy Approaches", Journal of Public Economics, 71, 235-245.

[8] Kimmel, S. (1992), "Effects of Cost Changes on Oligopolistic Profits", Journal of Industrial Economics, XL, 441-449.

[9] Milgrom, P. and J. Roberts (1990b), "Rationalizability, Learning, and Equilibrium in Games with Strategic Complementarities", Econometrica, 58, 1255-1278.

[10] Milgrom, P. and C. Shannon (1994), "Monotone Comparative Statics", Econometrica, 62, 157-180.

[11] Novshek, W. (1985), "On the Existence of Cournot Equilibrium", Review of Economic Studies, L II, 85-98.

[12] Rockafellar, T. (1970), Convex Analysis, Princeton University Press, Princeton.

[13] Seade, J. (1985), "Profitable Cost Increases and the Shifting of Taxation; Equilibrium Responses of Markets in Oligopoly", Unpublished manuscript, Warwick University.

[14] Suits, D.B. and R.A. Musgrave (1953), "Ad valorem and Unit Taxes Compared", Quarterly Journal of Economics, 67, 598-604.

[15] Topkis, D. (1978), "Minimizing a Submodular Function on a Lattice, Operations Research, 26, 305-321. 
[16] Topkis, D. (1998), Submodularity and Complementarity, Princeton University press, Princeton.

[17] Varian, H. (1992), Microeconomic Analysis, Norton, N.Y.

[18] Vives, X. (1990), "Nash Equilibrium with Strategic Complementarities", Journal of Mathematical Economics, 19, 305-321.

[19] Vives, X. (1999), Oligopoly Pricing: Old Ideas and New Tools, MIT Press, Cambridge, MA.

[20] Wicksell, K (1896), Finanztheoretische Untersuchungen, 15-21, Jena. 